\title{
THE SYMPTOMS OF DEPRESSION AND ANXIETY IN PATIENTS WITH DIABETIC FOOT TREATED WITH HYPERBARIC OXYGEN - PRELIMINARY RESCLTS
}

\author{
Agnieszka Koźmin-Burzyńska ${ }^{1)}$, Agnieszka Bratek $^{1)}$, Grzegorz Knefel $^{2)}$, Krzysztof Krysta $^{1)}$, Marek Kawecki $^{2)}$, \\ Irena Krupka-Matuszczyk ${ }^{3}$ \\ 1) Faculty and Clinic of Psychiatry and Psychotherapy of the Silesian Medical University of Katowice, Poland \\ ${ }^{2)}$ Dr. Stanisław Sakiel Burn Treatment Centre in Siemianowice Śląskie, Poland \\ 3) The Silesian Higher School of Medicine of Katowice, Poland
}

\section{ABSTRACT}

The aim of the study was to assess the level of anxiety and depressiveness in patients who had qualified for hyperbaric oxygen therapy (HBOT) for the treatment of ulcerative lesions in the lower limbs, occurring as a result of diabetic foot syndrome (DFS),. A total of 50 patients with type 2 diabetes and diabetic foot syndrome were enrolled to the study. All patients have received 30 sessions of HBOT. During the therapy blood glucose was measured and photographic documentation was carried out. All patients underwent the following procedures: medical history and socioeconomic interview, psychiatric examination, self-report and objective psychometric tests to measure anxiety and depressiveness. Based on the obtained results, we reported that patients with a greater degree of tissue damage had higher levels of depressive symptoms in the self-report tests as well as in the objective evaluation of the investigator. In terms of location of ulcerative lesions - the level of depressiveness was greater when the affected area included toes, and the level of anxiety was increased when it concerned the heel. Regarding other parameters, statistically significant correlations were not observed. Keywords: anxiety, depression, diabetic foot, hyperbaric oxygen, HBOT.

ARTICLE INFO

PolHypRes 2016 Vol. 54 Issue 1 pp. 23-30

ISSN: 1734-7009 elSSN: 2084-0535

DOI: $10.1515 /$ phr-2016-0003

Pages: 8 , figures: 0 , tables: 5

page www of the periodical: www.phr.net.pl

Publisher

Polish Hyperbaric Medicine and Technology Society

\section{Original article}

Delivery date:16.11.2015r.

Date of approval for print: 22.12 .2016 r. 


\section{INTRODUCTION}

Hyperbaric oxygen therapy (HBOT) is a still underestimated, advanced therapy that gives excellent results in a number of medical cases. Its application is multi-dimensional and the effects, for example in the case of difficult to heal wounds, are visible already at an early stage of treatment $[1,2]$. The effectiveness of HBOT is described inter alia in the treatment of stroke, rheumatism, Lyme disease, and diabetic foot syndrome (DFS), which is the subject of our study [3,4]. In the World Health Survey, a study conducted by the World Health Organization in 60 countries worldwide, it was found that depressive disorders coexisting with somatic diseases (e.g. diabetes, chronic obstructive pulmonary disease, asthma), significantly worsen the general somatic state through exacerbating the negative effects of the underlying disease [5].

Anxiety and depression are the subjects of many studies conducted among patients with chronic diseases, including diabetes [6]. On one hand, having diabetes creates many challenges for the patients due to the need of lifestyle change and the fear of complications and pain, which can lead to anxiety or depressiveness [8]. On the other hand, many researchers point to the relationship between emotional disturbances and difficulties in wound healing, which can lead to a vicious circle mechanism. Thus, hyperbaric oxygen not only speeds up the healing of ulcerative lesions resulting from complications of diabetes, but through visible results gives patients hope, which indirectly decrease the level of anxiety and depressiveness $[3,4]$.

The aim of this study was to answer the following research questions: is there a relationship between the duration of diabetes and of diabetic foot syndrome and the severity of depressive and anxiety disorders in diabetic patients? Does the location and extent of tissue damage impact the level of depressive and anxiety symptoms in the study group? and whether increased levels of $\mathrm{HbA} 1 \mathrm{c}$ correlate with the presence of depressive-anxiety symptoms?

\section{MATERIALS AND METHODS}

The study included 50 patients diagnosed with diabetic foot syndrome (DFS), 9 women (18\%) and 41 men $(82 \%)$ aged from 28 to 80 years old, treated at the Center for Burns Treatment in Siemianowice Slaskie from 2012 to 2014 [Table 1].

Statistical data regarding age, duration of diabetes and duration of diabetic foot syndrome

\begin{tabular}{llllll}
\hline Variable & Mean & Median & Minimum & Maximum & Variance \\
\hline Age & 59,06 & 60,00 & 28,00 & 81,00 & 107,77 \\
\hline Duration of diabetes (years) & 18,40 & 18,00 & 1,00 & 43,00 & 139,80 \\
\hline Duration of DFS (years) & 3,34 & 2,00 & 1,00 & 17,00 & 11,37 \\
\hline
\end{tabular}

The patients qualified for HBOT based on the results of percutaneous oximetry of the infected areas. Blood glucose was measured before and after the session, and HbA1c levels were determined before the first session.

The examinations were performed twice: the first time being on day one of the therapy (before entering to the hyperbaric chamber), and for the second time after the last session of HBOT. Affected areas were documented photographically before the first session and after the whole treatment cycle. All patients underwent a series of 30 therapy sessions with 100 percent oxygen at a pressure of 2.4 ATA, 90 minutes each, five times a week.

The study was based on patients' medical history, psychiatric examination, analysis of medical records, as well as the standardized clinical scales filled out by the physician: the Megitt-Wagner Scale, Hamilton Depression Rating Scale (HDRS), Mini-Mental State Examination (MMSE) and the questionnaire (initial and final) designed specifically for purposes of the research and patient self-report scales: Hospital Anxiety Depression Scale (HADS), Beck Hopelessness Scale (BHS) and the Patient Health Questionnaire 9 (PHQ-9). Patients were provided with complete anonymity and full confidentiality when performing the tests.

The following inclusion criteria were adopted:

- type 2 diabetes according to the ICD-10 criteria (category E 11)

- fulfillment of the criteria for diabetic foot syndrome,
- degree of tissue damage in the range of I to IV according to the Megitt-Wagner Scale,

- qualification for hyperbaric oxygen therapy,

- $\quad$ expressed conscious and voluntary consent to participate in the study,

- $\quad$ age - between 18 and 80 years old

- maintained emotional and intellectual contact

The exclusion criteria were as follows:

- lack of or withdrawal of written consent to participate in the research,

- not meeting the inclusion criteria,

- taking medication affecting significantly at least one of the studied parameters,

- cognitive impairment and / or known mental disorders that disturbs performing the examination,

- $\quad$ presence of serious physical or neurological disorders not related to diabetes that can significantly affect the mental state of participants,

- psychoactive substances dependence.

Research tools:

The Megitt-Wagner Scale - the classification of ulcers in diabetic foot created by Megitt and Wagner. It is based on an assessment of the wound depth and the extent of necrosis. The scale includes 6 degrees, where 0 indicates intact skin and 5 - necrosis of the entire foot 
$[9,10]$.

Patient Health Questionnaire 9 (PHQ-9) is a questionnaire consisting of nine fundamental questions and one additional question. The answer to each question is scored on a scale from 0 (never) to 3 (almost every day). The maximum number of points possible to gain is 27 and indicates the greatest severity of depression [11].

The Hospital Anxiety and Depression Scale (HADS) is an example of a self-assessment scale. It is used for screening of anxiety and depression features in hospitalized patients and outpatients. It contains 14 questions. The answers are evaluated by a 4-point Likert scale (range 0-3). In both subscales: anxiety (odd numbers) and depression (even numbers) results are summed up and their value is directly proportional to the severity of symptoms $[12,13]$.

The Beck Hopelessness Scale (BHS) is a selfreport tool for the assessment of patients' negative attitudes' towards the future, pessimism, hopelessness and loss of motivation. It consists of 20 statements, half of which describes the future as optimistic and half as pessimistic. The total score indicates a degree of hopelessness [14].

The Mini-Mental State Examination (MMSE) is a simple screening tool. Obtaining a score below the cut-off points to the need for further diagnostic tests to confirm or exclude dementia [15].

Hamilton Depression Rating Scale (HDRS) is a research tool used to objectively measure the severity of depressive symptoms. The scale used in the study consists of 21 questions to which the investigator answers based on an interview and examination conducted with the patient; assessment pertains to the current clinical state [16].
Questionnaire (preliminary and final) designed specifically for this research purposes. It includes questions on basic socio-demographic data (i.e. gender, age, education, occupation, marital status, place of residence), and data pertaining to the course and the beginning of diabetes and diabetic foot syndrome (i.e. treatment, severity and location of lesions). Patients also answered questions on their current emotional difficulties in view of the existing disease, the need and feasibility of obtaining support and whether they'd had contact with a psychiatrist either in the present or in the past (if so - whether or not psychopharmacotherapy was introduced).

Statistical analysis was performed using Statistica software version 10. In the case of quantitative variables, the normality of the distribution was tested by the Shapiro-Wilk test. If the variable was not subject to the normal distribution, the Mann-Whitney U test was used to assess the statistical significance of the variables' differences. When the distribution was normal, Student's t-test was used. In the case of qualitative variables, we used the chi2 test or Fisher's exact test, depending on the size of the group. Also, logistic regression analysis was performed. The level of statistical significance was $p$ $<0.05$.

\section{RESULTS}

To answer the research questions, we investigated the correlation between the level of depressive and anxiety symptoms in patients with diabetes treated with hyperbaric oxygen, and the following variables.: reported.

1. Duration of diabetes - no correlation was

2. Duration of diabetic foot syndrome - no correlation was reported (tab.2).

Correlation between the duration of diabetes and of DFS and the scales measuring the level of depressiveness and anxiety. Marked correlations are significant with $p<0,05$.

\begin{tabular}{lllll}
\multirow{2}{*}{ Variable } & \multicolumn{3}{l}{} \\
\cline { 2 - 5 } & BHS & HADS A & HADS D & Hamilton \\
\hline Duration of diabetes (years) & 0,20 & 0,19 & 0,20 & 0,23 \\
\hline Duration of DFS (years) & $-0,13$ & 0,03 & $-0,07$ & $-0,05$ \\
\hline
\end{tabular}

3. The degree of tissue damage - there are two correlates of the degree of tissue damage - to the PHQ-9, and Hamilton test. In both cases it was a directly proportional relationship - the greater the lesion, the higher the level of depressiveness. In both cases, the correlation was low, with a clear dependence between variables (tab.3).

Correlation between the extent of ulcerative lesions and psychometric tests. Marked correlations are significant with $p<0,05$.

\begin{tabular}{lllll}
\cline { 2 - 5 } \multicolumn{1}{c}{ Variable } & BHS & $\begin{array}{l}\text { HADS } \\
\text { A }\end{array}$ & HADS D & Hamilton \\
\hline $\begin{array}{l}\text { Extent of lesions (according to } \\
\text { Megitt-Wegner Scale) }\end{array}$ & 0,20 & 0,05 & 0,25 & 0,30 \\
\hline
\end{tabular}


To evaluate how independent variables explain the occurrence of depressive symptoms, two linear regression models were built - for PHQ-9 and for Hamilton. In both models, the degree of tissue damage turned out to be a statistically significant predictor of depressiveness levels measured with questionnaires. It the next step, the assumptions for the regression model were checked, in particular - the normality of the distribution of the regression residuals and the value of the Durbin-Watson statistics. In the studied models, there was no normal distribution of regression residuals. These models are therefore only partially matched, and it is necessary to take into account other predictors of depressiveness.

4. The location of ulcerative lesions:

- Toes II-V of the right foot and psychological tests - higher levels of depression (BHS and Hamilton) in patients with lesions within the aforementioned area.

- Toe I of the right foot and psychological tests higher levels of depression (PHQ-9) in patients with lesions within the aforementioned area.
- Toes II-V of the left foot and psychological tests higher levels of depression (BHS and Hamilton) in patients with lesions within the aforementioned area

- Toe I of the left foot, right and left metatarsal area and the right heel and psychological tests no significant differences were reported.

- Left heel and psychological tests - higher level of anxiety (HADS-A) in patients with lesions within the aforementioned area

- $\quad$ Right shin and psychological tests - no difference between comparison groups.

- Left shin and psychological tests - test not performed due to the lack of a comparison group

5. The level of glycosylated hemoglobin (HbA1c) (tab.5).

Correlations between psychological tests $\mathrm{HbA} 1 \mathrm{c}$. Marked correlations are significant with $\mathrm{p}<0,05$.

\begin{tabular}{ll}
\hline Variable & HbA1c $(\%)$ \\
\hline PHQ-9 & 0,14 \\
\hline BHS & 0,13 \\
\hline HADS A & $-0,06$ \\
\hline HADS D & 0,20 \\
\hline Hamilton & 0,08 \\
\hline
\end{tabular}

\section{DiscuSSION}

Chronic illness is a prolonged or recurrent condition. It may be asymptomatic for many years, and symptoms can appear gradually. In the case of diabetes, the factors that play the most important role are the time of diagnosis and patient's compliance to recommendations on prevention, including diet and healthiness of lifestyle.

The effectiveness of therapy of chronic diseases largely depends on the age, circumstances and the general condition of the patient, but not only. An increasing number of research studies point to the impact of mental state on the wound healing process (for example in the course of DFS) $[17,18]$.

We also reported a relationship between depressiveness as measured with self-assessment and objective tools, and the severity of ulcerative lesions related to diabetic foot syndrome. It was a directly proportional relationship - subjects who had greater degrees of tissue damage (according to the MegittWagner Scale) declared a higher level of depressive symptoms in the Patient Recovery Questionnaire, and received more points in the objective assessment of the investigator (Hamilton Depression Scale).

With regards to the location of ulcerative lesions, we observed a positive correlation between the subjective and objective level of depressiveness and lesions located in the toes II-V of both feet and the right toe I. Respondents whose affected areas included the heel, scored higher in self-assessment tests of anxiety.
During the interviews carried out during the tests, patients repeatedly stressed the symbolic importance of toes, they claimed to be "not that sick, because I still have toes" and presented anxiety symptoms associated with their loss. Low mood and sadness was visible in most patients with ulcers located in the metatarsal area - "because it means, you know, that it is getting worse."

There was no significant relationship between HbA1c and the severity of depressive/anxiety symptoms. The lack of correlation may be the result of using too small a study group, which will be taken into account during future research. In the published studies on similar subject, the high level of satisfaction with treatment was correlated with low levels of HbA1c and normal weight [19].

Other authors have also observed the relationship between gender, glycemic control (HbA1c), and the prevalence of depressiveness and anxiety [20].

\section{Conclusions}

The present study provides a holistic view on patients treated in a hyperbaric chamber due to a somatic illness and assess the impact of emotions on the process of therapy.

We hope that it will provoke a discussion on attempts to apply HBOT to the treatment of psychiatric disorders, i.e. depression. Literature on this subject is scarce and found so far only in the English language $[21,22]$. 
However, still little is known about the relationship between symptoms of depression and anxiety in patients after the treatment in a hyperbaric chamber, and all reports (as well as our work) apply to indirect effects of treatment of depressiveness and anxiety secondary to somatic disorders.

\section{BIBLIOGRAPHY}

1. AI-Waili NS, Butler GJ, Beale J et al. Hyperbaric oxygen in the treatment of patients with cerebral stroke, brain trauma, and neurologic disease. Adv Ther 2005; 22: 659-678.

2. Al-Waili NS, Butler GJ, Beale $\mathrm{J}$ et al. Hyperbaric oxygen and malignancies: a potential role in radiotherapy, chemotherapy, tumor surgery and phototherapy. Med Sci Monit 2005; 11: RA279-RA289;

3. Cao H, Ju K, Zhong L, Meng T. Efficacy of hyperbaric oxygen treatment for depression in the convalescent stage following cerebral hemorrhage. Exp Ther Med. 2013 Jun;5(6):1609-1612;

4. Yan D, Shan J, Ze Y, Xiao-Yan Z, Xiao-Hua H. The effects of combined hyperbaric oxygen therapy on patients with post-stroke depression J Phys Ther Sci. 2015 May;27(5):1295-7;

5. Moussavi S, Chatterji S, Verdes E. et al. Depression, chronic diseases, and decrements in health: results from the World Health Surveys. Lancet, 2007, 370, 851-58;

6. van Dooren FEP, Nefs G, Schram MT, Verhey FRJ, Denollet J, et al. (2013) Depression and Risk of Mortality in People with Diabetes Mellitus: A Systematic Review and Meta-Analysis. PLoS ONE 8(3): e57058;

7. Cole-King A, Harding KG. Psychological factors and delayed healing in chronic wounds. Psychosom Med. 2001 Mar-Apr;63(2):216-20;

8. Tatoń J. Guidebook for individuals with type 2 diabetes not requiring insulin treatment. Wydawnictwo Lekarskie PZWL, Warsaw, 2002;

9. Megitt B. Surgical management of the diabetic foot. Br. J. Hosp. Med. 1976;227,216;

10. Wagner W. The dysvascular foot: a system for diagnosis and treatment. Foot Ankle 1981;2,64;

11. Tomaszewski K, Zarychta M, Bieńkowska A, Chmurowicz E, Nowak W, Skalska A. Validation of the Polish language version of Patient Health Questionnaire-9 in the population of hospitalised elderly. Psychiatria Polska 2011, vol. XLV, no. 2 pp. 223-233;

12. Wichowicz H, Wieczorek D. Screening tests for post-stroke depression with the use of Hospital Anxiety and Depression Scale (HADS) Psychiatria Polska 2011, vol. XLV, no. 4, pp. 505-514

13. Łoza B. How to simultaneously measure anxiety and depression? Hospital Anxiety and Depression Scale. Wiadomości Psychiatryczne, vol. 14, no. 4/2011, pp. 225-230;

14. Beck AT, Weissman A, Lester D, Trexler L. The measurement of pessimism: the hopelessness scale. J Consult Clin Psychol. 1974 Dec;42(6):861-5;

15. Kotapka-Minc S. The importance of neuropsychological examination in the diagnosis of dementia. Polski Przeglad Neurologiczny 2007, vol. 3 , 2, pp. 61-68;

16. Hamilton M. A rating scale for depression. J. Neurol. Neurosurg. Psychiatry. 1960; 23: 56-62;

17. Schmitt J, Ford D. Understanding the relationship between objective disease severity, psoriatic symptoms, illness-related stress, health-related quality of life and depressive symptoms in patients with psoriasis - a structural equations modeling approach General Hospital Psychiatry 29 (2007) 134-140;

18. Menzies V. Depression and Burn Wounds. Archives of Psychiatric Nursing, Vol. XIV, No. 4;

19. Bradley C, Lewis S. Measures of psychological well-being and treatment satisfaction developed from the responses of people with tablet-treated diabetes. Diabet. Med., 1990, 7, 5, 445-451

20. Lloyd E, Dyer H, Barnett A. Prevalence of symptoms of depression and anxiety in a diabetes clinic population. Diabet. Med., 2000, 17, 198-202;

21. Cao H, Ju K, Zhong L, Meng T. Efficacy of hyperbaric oxygen treatment for depression in the convalescent stage following cerebral hemorrhage. Exp Ther Med. 2013 Jun; 5 (6):1609-1612;

22. Yan D, Shan J, Ze Y, Xiao-Yan Z. The effects of combined hyperbaric oxygen therapy on patients with post-stroke depression. J Phys Ther Sci. 2015 May;27(5):1295-7.

\section{Doctor Agnieszka Koźmin-Burzyńska}

Faculty and Clinic of Psychiatry and Psychotherapy of the Silesian Medical University of Katowice

$$
\text { ul. Ziołowa 45-47, 40- } 635 \text { Katowice, }
$$

$$
\text { e-mail: kozmin@mp.pl }
$$

